\title{
An Extraction of the Skewing Factor from DESY-HERA Data
}

\author{
Laurent Favart, \\ I.I.H.E., Université Libre de Bruxelles, 1050 Brussels, Belgium \\ Magno V. T. Machado, \\ Universidade Federal do Pampa - UNIPAMPA, Campus Bagé. \\ Rua Carlos Barbosa, CEP 964000-970. Bagé, RS, Brazil \\ and Laurent Schoeffel \\ CE Saclay, DAPNIA-SPP, 91191, Gif-sur-Yvette, France
}

Received on 3 October, 2006

\begin{abstract}
In this contribution we present recent investigation on the extraction of the skewing factor, defined as the ratio of the imaginary parts of the amplitudes $\operatorname{Im} \mathcal{A}\left(\gamma^{*} p \rightarrow \gamma^{*} p\right) / \operatorname{Im} \mathcal{A}\left(\gamma^{*} p \rightarrow \gamma p\right)$. This factor is extracted from the data using the recent DVCS and the inclusive inelastic cross section measurements at DESY-HERA. We compare the results to the theoretical predictions for NLO QCD and the color dipole approach.
\end{abstract}

Keywords: Deeply virtual Compton scattering; High energy physics; Small- $x$ physics; Saturation models

\section{INTRODUCTION}

The study of hard exclusive reactions in the Bjorken limit is crucial to obtain information on parton dynamical correlations in the nucleon. It is well known that the cross section of hard scattering processes can be described as the convolution of parton distributions (PDFs) and the cross sections of hard subprocesses computed at parton level using perturbative QCD. The usual PDFs, obtained from inclusive experimental data, is the diagonal element of an operator in the Wilson operator product expansion (OPE). On the other hand, there is a set of exclusive reactions which are described by the off-diagonal elements of the density matrix, where the momentum, helicity or charge of the outgoing target are not the same as those of the corresponding incident particle. Examples of such reactions are the virtual photon Compton scattering (DVCS) $\left(\gamma^{*} p \rightarrow \gamma p\right)$ and the vector meson electroproduction $\left(\gamma^{*} p \rightarrow V p\right)$. In these cases, the difference with the inclusive case is the longitudinal components of the incoming and outgoing proton momentum, which depend on the photon virtuality $Q^{2}$ and the $\gamma^{*} p$ center of mass energy $W$.

The recent data from DESY ep collider HERA on exclusive diffractive virtual Compton process[1-3] at large $Q^{2}$ becomes an important source to study the partons, in particular gluon, inside the proton for non-forward kinematics and its relation with the forward one. A considerable interest of the DVCS process comes from the particular access it gives to these generalized parton distributions (GDP) through the interference term with the Bethe-Heitler process. In what follows, we present the recent results on the experimental extraction of the skewing correction factor $R$ [4], which it was performed by computing the ratio of the imaginary parts of the amplitudes for the DIS and DVCS reactions. This knowledge enables us to gain insight into the skewedness correction to the non-forward observables.

\section{EXPERIMENTAL EXTRACTION OF SKEWING FACTOR}

The optical theorem states that the leading DIS process of a single virtual photon exchange can be viewed as identical to the forward elastic scattering of a virtual photon from the proton. This scattering, known as the forward Compton scattering process, is the same as a DIS process with its mirrored reaction and relates the total cross section $\sigma_{t o t}\left(\gamma^{*} p \rightarrow X\right)$ with the imaginary part of the forward amplitude $\operatorname{Im} A\left(\gamma^{*} p \rightarrow \gamma^{*} p\right)$. The forward Compton scattering $\gamma^{*}(q) p(p) \rightarrow \gamma^{*}(q) p(p)$ can be generalized to the non forward case (at non-zero angles): $\gamma^{*}(q) p(p) \rightarrow \gamma^{(*)}\left(q_{2}\right) p\left(p_{2}\right)$ with $q_{2} \neq q$ and $p_{2} \neq p$ and being experimentally accessible through the so called Deeply Virtual Compton Scattering (DVCS) process, where a real photon is observed in the final state.

Lets now address the question of the extraction of the skewing correction using the available data on DIS and DVCS. This can be performed by computing the ratio of the imaginary parts of the amplitudes for these reactions. This knowledge will enable us to gain insight into the skewedness correction to the non-forward observables. Lets define a basic quantity giving an overall measurement of the skewing properties, which includes both the non-forward kinematics and the non-diagonal effects. Namely, we set the ratio between the imaginary parts of the DIS and DVCS (forward) scattering amplitudes at zero momentum transfer:

$$
R \equiv \frac{\left.\operatorname{Im} \mathcal{A}\left(\gamma^{*}+p \rightarrow \gamma^{*}+p\right)\right|_{t=0}}{\left.\operatorname{Im} \mathcal{A}\left(\gamma^{*}+p \rightarrow \gamma+p\right)\right|_{t=0}},
$$

where $t$ is the square of the four-momentum exchanged at the proton vertex.

The scattering amplitude for the DIS process can be directly obtained from the DIS cross section and experimentally measured at DESY-HERA, that is $\operatorname{Im} \mathcal{A}\left(\gamma^{*} p \rightarrow \gamma^{*} p\right) \sim$ $\sigma_{t o t}\left(\gamma^{*} p \rightarrow X\right)$. In fact, the DIS amplitude can be written down 


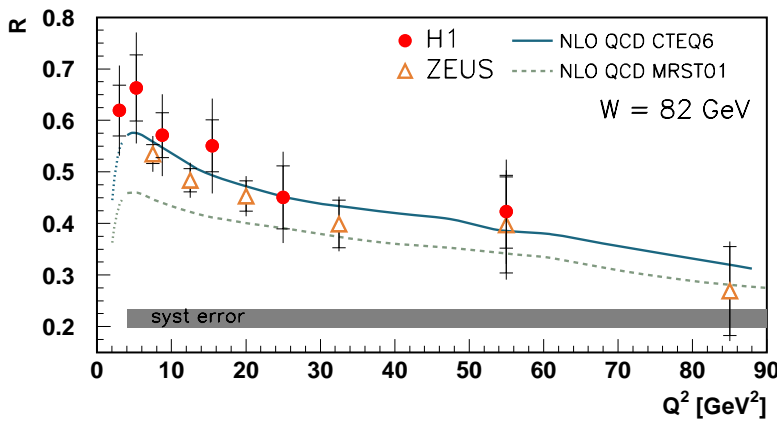

(a)

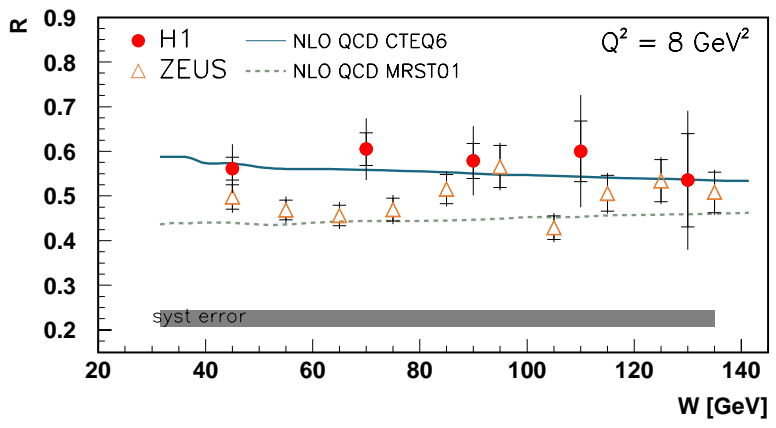

(b)

FIG. 1: (a) The skewing factor $R$ as a function of $Q^{2}$ at $W=82 \mathrm{GeV}$ [4]. The points correspond to the $R$ extraction applied to DVCS measurements of H1 (bullets) and ZEUS (triangles). The curves represent $R$ extracted from Freund et al. prediction based on MRST 2001 and CTEQ6 PDFs. (b)The skewing factor $R$ as a function of $W$ at $Q^{2}=8 \mathrm{GeV}^{2}$.

using the usual $\mathrm{pQCD}$ fits for the proton structure function, $\sigma_{\text {tot }}^{\gamma^{*} p}=\left(4 \pi^{2} \alpha / Q^{2}\right) F_{2}^{p}\left(x, Q^{2}\right)$. The DVCS scattering amplitude can be obtained from the recent measurements on the Deeply Virtual Compton Scattering cross section. In this case, the $t$ dependence of the amplitude can be assumed to be factorised out and parameterized as an exponential, $\propto \exp (-b|t|)$, and the total DVCS cross section can be related to the corresponding amplitude at $t=0$. The typical contribution of the real part of amplitude for the kinematic window available at HERA is of the order of $20 \%$. In particular, to a good approximation, $\eta$ can be calculated using dispersion relations to $\eta \simeq \tan (\pi \lambda / 2)$, where $\lambda=\lambda\left(Q^{2}\right)$ is the effective power of the Bjorken $x$ dependence of the imaginary part of the amplitude. For an estimate of the real part contribution we use the effective power for the inclusive deep inelastic reaction taken from Ref. [5].

Considering the calculation discussed above, we can rewrite the skewing factor as a function of the total cross sections for DIS and DVCS. The factor reads as,

$R=\frac{\sigma\left(\gamma^{*} p \rightarrow X\right) \sqrt{\left(1+\eta^{2}\right)}}{4 \sqrt{\pi b \sigma\left(\gamma^{*} p \rightarrow \gamma p\right)}}=\frac{\sqrt{\pi^{3}} \alpha}{Q^{2}} \frac{F_{2}^{p}\left(x, Q^{2}\right) \sqrt{\left(1+\eta^{2}\right)}}{\sqrt{b \sigma\left(\gamma^{*} p \rightarrow \gamma p\right)}}$

Main theoretical uncertainties come from the $b$ slope and from the estimate of the real part contribution. In our further calculation, one uses the $b$ value extracted from the recent measurements of the $t$-dependence of DVCS data.

To extract the factor $R$ factor, Eq. (2), we use recent DVCS measurements at HERA [2,3] and the DIS cross cross section is obtained using the pQCD fits of the $F_{2}^{p}$ structure function. The factor $R$ is shown as a function of $Q^{2}$ in Fig. 1-a and as a function of energy $W$ in Fig. 1-b. The inner error bars represent the statistical error. The full error bars is the quadrature sum of the statistical, systematic and normalization (precision of the $b$ measurement of $\mathrm{H} 1$ ) uncertainties. A $Q^{2}$ dependence is observed, decreasing from $R \simeq 0.7$ for $Q^{2} \simeq 2 \mathrm{GeV}^{2}$ down to $R \simeq 0.3$ for $Q^{2} \simeq 85 \mathrm{GeV}^{2}$. Qualitatively, the data seem to be consistent with a dependence like $R \propto 1 / \log Q^{2}$. An almost flat $W$ dependence is observed within the present precision. This feature can be easily understood by inspecting Eq.
(2), since the $W$ dependence of both the DIS and DVCS cross section is power-like having a proportional effective power. Namely, $\sigma_{\text {DIS }} \propto W^{2 \lambda}$ and $\sigma_{\text {DVCS }} \propto W^{4 \lambda}$. The mean value $R \simeq 0.5$ is consistent with its early theoretical estimates using the aligned jet model and the color dipole saturation model $[6,7]$.

Lets compare the results extracted from experimental data to different theoretical predictions. First, we contrast them with the perturbative QCD approach at NLO accuracy. This prediction is dependent on the Generalised Parton Distributions (GPD), which have been parameterized in Ref. [8] and applied to describe the recent DVCS data $[9,10]$. The second theoretical approach $[6,7]$ is given by the color dipole approach, where the basic building blocks are the photon wavefunctions and the dipole cross section. The non-forward kinematics is encoded by the wavefunctions whereas the offdiagonal effects can be built-in in the parameterizations for the dipole-target cross section.

The DVCS cross section has been calculated at NLO in perturbative QCD by Freund and McDermott $[9,10]$ using two different GPD parameterizations [8]. The MRST2001 and CTEQ6 parameterizations of the PDFs are used in the DGLAP region and polynomial form is used in the ERBL region, ensuring a smooth continuation between the two regions. Both the skewing $(\xi)$ and the $Q^{2}$ dependence are generated dynamically. The $t$ dependence is factorised out and assumed to be $e^{-b|t|}$. In its original form, the approach above used a $Q^{2}$-dependent $b$ slope. We have verified that the recent data on DVCS [3] can be reasonably described using a fixed $b$ slope.

Applying the same method presented here (i.e. using the same $F_{2}^{p}$ ) for the inclusive cross section, the values obtained are shown as a function of $Q^{2}$ in Fig. 1-a and as a function of $W$ in Fig. 1-b. The value of $b=6.02 \pm 0.35 \pm 0.39 \mathrm{GeV}^{-2}$ measured by $\mathrm{H} 1$ [3] has been used in all predictions. A good agreement between these predictions and the data points is found, describing well the absolute value of $R$ and its kinematic dependences. The NLO calculation presents no $W$ dependence. The result normalization is strongly dependent on 


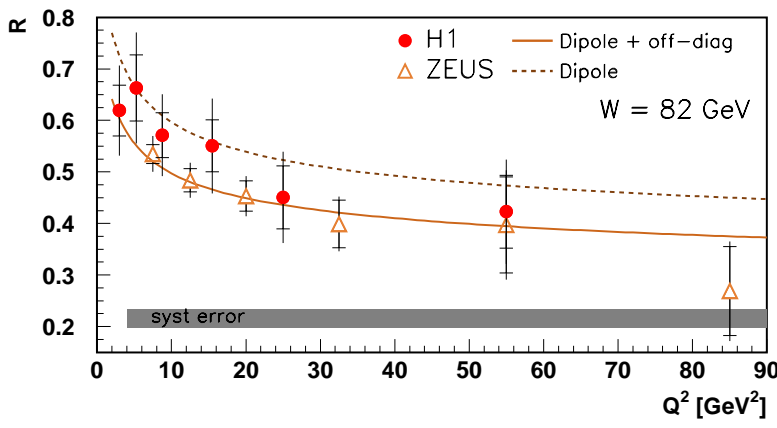

(a)

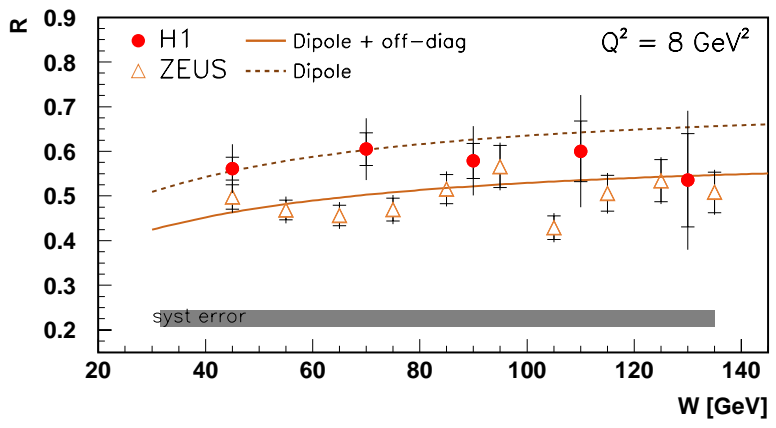

(b)

FIG. 2: (a) The skewing factor $R$ as a function of $Q^{2}$ at $W=82 \mathrm{GeV}$ [4]. The curves represent the theoretical predictions of the color dipole approach (see text) with (solid) and without (dashed) applying the off-diagonal skewing factor $R_{g}$. (b) The skewing factor $R$ as a function of $W$ at $Q^{2}=8 \mathrm{GeV}^{2}$

the choice for the gluon distribution and the deviations seem to be amplified at lower $Q^{2}$. Concerning the behavior on $Q^{2}$, it seems that for $Q^{2}$ values below $\simeq 4 \mathrm{GeV}^{2}$ the DGLAP evolution starting at $Q_{0}^{2}=1 \mathrm{GeV}^{2}$ has too little phase space to fully generate the gluon distributions (dotted part of the curves in Fig. 1-a).

In the color dipole approach, the imaginary part of the DIS (or DVCS) amplitude at $t=0$ is expressed in the simple way [6]. The non-forward kinematics for DVCS is encoded in the color dipole approach through the different weight coming from the photon wavefunctions. The off-diagonal effects, which affect the gluon and quark distributions in the pQCD approaches, should be included in the parameterization of the dipole cross section. At the present stage of the development of the color dipole formalism, we have no accurate theoretical arguments on how to compute skewedness effects from first principles. In Ref. [7] an estimate of the skewedness for the dipole cross section has been performed using the approximation of Ref. [11] where the ratios of off-diagonal to diagonal parton distributions are computed. The ratio is larger for singlet quarks than for gluons.

In the color dipole picture, the DVCS cross section is driven mainly by gluonic exchanges. In our numerical computations, we use $\lambda=\lambda\left(Q^{2}\right)$ as obtained from the DVCS scattering amplitude and the skewedness effect is given by multiplying the original (without skewedness) total cross section by the factor $R_{g}^{2}\left(Q^{2}\right)$. Using this color dipole prediction (with DGLAP evo- lution and off-diagonal skewing factor $R_{g}$ ), one can again extract the $R$ factor, as shown as a function of $Q^{2}$ in Fig. 2-a and as a function of $W$ in Fig. 2-b. A good agreement with points extracted from the data is found. The curve obtained without applying the off-diagonal skewing factor $R_{g}$ is also presented, enabling one to isolate the two effects: the non-forward kinematic and the off-diagonal gluon distributions. Furthermore, the off-diagonal effects contribute mostly for the overall normalization of the skewing factor whereas the behavior on $Q^{2}$ seems to be driven by the non-forward kinematic effect. This prediction exhibits a moderate increasing of $R$ with $W$ which is not present in the NLO prediction.

As a summary, the skewing factor $R$, defined as the ration of the imaginary parts of the DIS and DVCS amplitudes has been extracted from experimental data. In its determination one uses the recent DVCS and the inclusive inelastic cross section measurements at DESY-HERA. The main theoretical uncertainties come from the $b$ slope and from the estimate for the real part contribution. One founds a $Q^{2}$ dependence qualitatively consistent with the form $R \propto 1 / \log Q^{2}$. No $W$ dependence is observed within the current precision. The mean value $R \simeq 0.5$ is consistent with previous theoretical estimates in the jet aligned model and color dipole picture. The extracted $R$ is contrasted to theoretical predictions of NLO QCD and color dipole approaches.
[1] C. Adloff et al. [H1 Collaboration], Phys. Lett. B 517, 47 (2001).

[2] S. Chekanov et al. [ZEUS Collaboration], Phys. Lett. B 573, 46 (2003).

[3] C. Aktas et al. [H1 Collaboration], Eur. Phys. J. C 44, 1 (2005).

[4] L. Favart, M. V. T. Machado and L. Schoeffel, arXiv:hep$\mathrm{ph} / 0511069$.

[5] C. Adloff et al. [H1 Collaboration], Phys. Lett. B 520, 183 (2001), [hep-ex/0108035].
[6] L. Favart and M. V. T. Machado, Eur. Phys. J. C 29, 365 (2003).

[7] L. Favart and M. V. T. Machado, Eur. Phys. J. C 34, 429 (2004).

[8] A. Freund, M.F. McDermott, and M. Strikman, Phys. Rev. D 67, 036001 (2003).

[9] A. Freund and M.F. McDermott, Phys. Rev. D 65, 091901 (2002).

[10] A. Freund and M.F. McDermott, Phys. J. C 23, 651 (2002).

[11] A. G. Shuvaev et al., Phys. Rev. D 60, 014015 (1999). 